\title{
JORNADAS DE ESTUDIO SOBRE ADMINISTRACION Y FUNCION PUBLICA EN EL ESTADO AUTONOMICO
}

\author{
por \\ Agustín Pérez Barrio \\ Becario del I.V.A.P.
}

SUMARIO: I. INTRODUCCION.-II. PRIMERA SESION.-III. SEGUNDA SESION.-IV. TERCERA SESION.-V. CLAUSURA.VI. CONCLUSIONES FINALES.

\section{INTRODUCCION}

El reto pendiente de construir una Administración pública autonómica eficaz, que supere los defectos tradicionales de la Administración pública española, así como la necesidad de una reflexión sobre el tema en el actual proceso legislativo sobre Función Pública existente en la Comunidad Autónoma vasca, han llevado a la organización de estas Jornadas por el Instituto Vasco de Administración Pública y la Facultad de Derecho de la Universidad del País Vasco.

La celebración de las mismas ha tenido lugar en el Salón de Grados de la Facultad de Derecho de San Sebastián, en reuniones de mañana y tarde, los dias 23,24 y 25 de octubre, habiéndose dado cita junto con los ponentes más de doscientos participantes prove- 
nientes del ámbito universitario, profesional y político, presentándose tres comunicaciones $(*)$.

La intención de esta crónica es realizar una aproximación al contenido de las ponencias y a sus autores dada la próxima publicación de las mismas por el Instituto Vasco de Administración Pública, que facilitará su amplio examen y crítica.

Las Jornadas se estructuraron en tres reuniones: «El marco general», «Experiencias puntuales» $\mathrm{y}$ «Experiencias funcionariales en Euskal-Herria».

\section{PRIMERA SESION}

La sesión inaugural fue presidida por Elisabete BIZKARRALEGORRA OTAZUA, Directora del Instituto Vasco de Administración Pública, quien tras la bienvenida y salutación a los presentes expresó la esperanza de que estas Jornadas puedan servir como punto de referencia a los distintos poderes públicos.

Tras la presentación de los ponentes a cargo de José Manuel CASTELLS ARTECHE, se comenzó con la ponencia titulada: «Administración pública y burocracia en el Estado español en la década de los ochenta», expuesta por el Catedrático de Derecho Administrativo de la Universidad de Alcalá, Alejandro NiETo.

Las líneas fundamentales de la misma fueron las siguientes:

Calificando como estériles las etapas de tardofranquismo y la transición, previas a la década de los ochenta, se preguntó si en ésta ha sucedido algo en la Administración en general, dando una respuesta negativa.

A continuación señaló el inmovilismo o aferramiento a las estructuras anteriores y la improvisación, como notas características de esta década, apuntando igualmente los defectos básicos condicionantes de la política funcionarial de la misma:

En primer lugar, la ruptura entre el programa del PSOE y la posición del Gobierno socialista, destacando el desplazamiento del poder de decisión en materia de Administración y Función Pública al Ministerio de Economía y Hacienda.

$\left(^{*}\right)$ Las comunicaciones presentadas fueron las siguientes:

- "Los Gabinetes", por el señor JIMENEZ ASENSIO.

- "Autonomia parlamentaria y Función Pública", por los señores Aguiló Lucia y Visiedo Mazón y la señora Sevilla Merino.

- "Organización de la Función Pública local», por el señor ZuRITA. 
En segundo lugar, el aislamiento total del Ministerio de Presidencia respecto del propio partido, de la Administración y de los sindicatos.

En tercer lugar, la ausencia de un programa de acción.

Situando el análisis de la política funcionarial en la década de los ochenta dentro del marco anterior, destacó los caracteres esenciales de esta política. Fundamentalmente los siguientes:

1) Arbitrismo comparable al del siglo XVII, anacronismo o tercermundismo.

2) Cesión ante presiones de toda índole: del propio partido, internas y externas.

3) Actuación a través del mecanismo estímulo-respuesta.

4) La posposición de los problemas espinosos.

5) Predominio del Ministerio de Economía y Hacienda sobre el Ministerio de Presidencia, fundamentalmente en materia de retribuciones.

6) Revanchismo vacilante o ambiguo contra los funcionarios y los Cuerpos.

7) Irrealismo. La Ley 30/1984, de Medidas para la Reforma de la Función Pública, no tiene nada que ver con la realidad.

8) La megalomanía fácil. La Ley $30 / 1984$ pretende abarcar la Administración estatal, autonómica y local, pero en realidad se va a aplicar a un colectivo reducido de funcionarios.

Como resumen de su exposición afirmó que no se ha hecho nada sustancial, quedando todo en anuncios e irregularidades. Asimismo señaló el problema de adaptación de las Comunidades Autónomas a la Ley 30/1984, Ley irreal, contradictoria y ambigua que no deja actuar a éstas, por lo que va a ser necesario trabajar y discurrir para traducir sus disposiciones en algo real; calificando, por último, el lastre de esta Ley como terrible.

La segunda ponencia, titulada «La Ley de Medidas para la Reforma de la Función Pública: su incidencia en la estructuración del personal de las Comunidades Autónomas», fue presentada por el Catedrático de Derecho Administrativo de la Universidad Complutense, Tomás Ramón FERNÁNDEZ.

En primer lugar, el ponente relacionó el objetivo tópico de reforma de la Administración que pretende esta Ley con el proceso de reforma de 1964-65, proceso a consecuencia del cual la Administra- 
ción ganó en calidad respecto al personal que la servía y a los procedimientos, desapareciendo los principales vicios de nuestra Administración no obstante la permanencia de las bases estructurales corporativas.

A continuación, se centró en la incidencia de esta Ley en la estructuración del personal de las Comunidades Autónomas constatando la inexistencia de un modelo de Función Pública, pero intentando averiguarlo a través de la interpretación de la Exposición de Motivos de la citáda Ley y el análisis de su articulado.

El esquema argumental fue el siguiente:

En primer término y partiendo del objetivo principal de esta Ley según su Exposición de Motivos: "Suprimir los obstáculos que una legislación vieja anterior a la Constitución opone al desarrollo del Estado autonómico», intentó descubrir estos obstáculos en el análisis de la regulación considerada como básica por la Ley respecto a los Cuerpos, las reglas de selección de los funcionarios, el sistema de provisión de puestos de trabajo, la carrera administrativa, las situaciones, las retribuciones, la jubilación forzosa y las incompatibilidades; intento que resultó en vano, puesto que estas materias no constituyen tales obstáculos. La consecuencia es la imposibilidad para el intérprete de ver el obstáculo de la regulación anterior y de apreciar el objeto principal de la Ley.

Tras este análisis, destacó el carácter y signo centralizador de dicha Ley, ya que todo lo importante sobre la Función Pública se regula con carácter general y básico, sin apenas margen para su regulación autonómica.

Seguidamente examinó los objetivos secundarios que pretende la Ley $30 / 1984$.

Respecto al primero: "Afrontar de manera realista el problema de la carrera administrativa", destacó la irrealidad del sistema al estar montadas las garantías de igualdad sobre las retribuciones básicas, siendo las realmente importantes las retribuciones complementarias en las que no rige esta garantía.

Como consecuencia, el realismo pretendido consiste en consagrar la máxima discrecionalidad en materia retributiva, discrecionalidad observada igualmente en el sistema de provisión de puestos de trabajo.

Respecto al segundo: "La racionalización de la estructura de Cuerpos y Escalas de la Administración pública como medio nece- 
sario para acercar la Administración a los ciudadanos», advìrtió la imposibilidad de adivinar tal objetivo en el articulado de la Ley.

Sin oponerse totalmente a su supresión y refundición de Cuerpos, criticó la operatividad de ciertos prejuicios sobre la «bestia negra» de los Cuerpos especiales y la pérdida para la Administración de. muchos aspectos positivos incorporados por un auténtico espíritu de servicio existente en muchos de los grandes Cuerpos liquidados por esta Ley.

Respecto al tercer objetivo secundario: «Adaptar nuestra Función Pública a las normas vigentes en los países de nuestro entorno", declaró que lo realizado por la Ley 30/1984 no tiene nada que ver con lo pretendido, pues ésta no tiene ni una articulación clara ni unos principios inspiradores.

Este proceso interpretativo le llevó a la afirmación de la inexistencia de un modelo de Función Pública y la existencia de un contexto de inseguridad jurídica que pone a los funcionarios en una situación peligrosa en la que domina su resignación y resistencia pasiva.

Por último, examinó la perspectivas futuras que se derivan de esta Ley. En este sentido constató varios hechos:

- A mayor discrecionalidad, mayor inseguridad jurídica.

- Disminución de incentivos para entrar y permanencer en la Administración pública.

- Desmoralización y pasividad de los funcionarios.

- Tendencia a la huida, bien vía excedencia o vía Comunidad Autónoma.

Para el ponente, la incidencia positiva de estos hechos en la estructuración del personal de las Comunidades Autónomas radica en la posible capitalización, no sin esfuerzo, del desmoralizamiento de la Función Pública estatal por las Comunidades Autónomas.

La incidencia negativa se manifiesta en el escaso hueco que deja la Ley para la regulación autonómica y en el impedimento que supone la misma a las Comunidades Autónomas para que busquen un modelo de Función Pública inexistente a nivel estatal, por lo que la situación actual puede calificarse de paréntesis, aventurándose un futuro incierto.

La tercera ponencia, titulada «La Función Pública local en la Ley de Bases del Régimen local», fue expuesta por el Profesor Titular de Derecho Administrativo de la Universidad de Oviedo, Raúl BOCANEGRA SIERRA. 
El contenido de la exposición se centró en el estudio del Título VII de la Ley 7/1985, de 2 de abril, Reguladora de las Bases de Régimen local, dedicado al personal al servicio de las Entidades locales, aunque excluyó del tratamiento al personal laboral y eventual.

La exposición del tema así encuadrado se efectuó dentro del contexto formado por el carácter básico de esta Ley, desarrollo del artículo 149, 1, 18, de la Constitución, el principio de autonomía de las Corporaciones locales, la Ley 30/1984 de Medidas para la Reforma de la Función Pública y los pronunciamientos del Tribunal Constitucional sobre diferentes extremos de la Función Pública local.

El análisis del Título VII se dividió en tres partes:

1) La referencia que hace la Ley de Bases de Régimen local a los funcionarios locales con Habilitación nacional.

2) La referencia a los funcionarios propios de las Corporaciones locales.

3) La referencia a las disposiciones comunes a los funcionarios de carrera.

Respecto a la primera parte, el ponente apuntó la aceptación por la Ley de Bases de Régimen local de la opción constitucional por el sistema funcionarial para establecer el régimen jurídico básico de los servidores públicos, así como de la reserva de determinadas funciones públicas a unos funcionarios con Habilitación de carácter nacional.

Esta reserva, realizada por el artículo 92, 3, de la Ley de Bases de Régimen local, fue considerada como uno de los rasgos definidores de la configuración de la Función Pública local realizada por esta Ley, observando algunos problemas importantes derivados de tal reserva.

En primer lugar, se suscita la cuestión de si la determinación de lo básico, que corresponde al Estado, puede alterar el orden competencial establecido por la Constitución y los Estatutos.

La respuesta la obtiene de las ideas que extrae de la Sentencia del Tribunal Constitucional de 7 de abril de 1983, para la cual la determinación de las Bases, función atribuida por la Constitución al Estado con gran amplitud y completa libertad - según el ponente-, supone la asignación a la legislación básica de una función determinadora indirecta de las competencias de las Comunidades Autónomas, por lo que el establecimiento del carácter básico de esta 
existencia de funcionarios locales con Habilitación nacional no supone una violación de las normas estatutarias, sino un ejemplo de la función delimitadora de la legislación básica.

En segundo lugar, se suscita si la reserva citada es contradictoria con la reforma global operada por la Ley $30 / 1984$, al venir a sustituir ésta la idea de función reservada al Cuerpo por la idea de puesto de trabajo.

Relacionando el contenido del artículo 15, 2, de la Ley 30/1984, que no tiene carácter básico, con lo preceptuado en el artículo 101 de la Ley de Bases de Régimen local, llega a la conclusión de que no se produce tal contradicción.

En tercer lugar; se plantea si la selección de estos funcionarios por una instancia diversa a la municipal supone una infracción al principio de autonomía local.

El tema lo consideró resuelto en la Ley de Bases de Régimen - local al entender que la selección de estos funcionarios no afecta al principio constitucional de autonomía local.

Respecto a la selección, formación y provisión de puestos de trabajo de estos funcionarios, señaló la finalidad de formación unitaria y homogénea pretendida por el artículo 98 de la Ley de Bases de Régimen local, advirtiendo, por una parte, la clara separación entre la habilitación, la atribución de idoneidad para desempeñar un cargo y los concursos para la provisión de puestos de trabajo, $\mathrm{y}$, por otra, la excepción a este sistema para el País Vasco producida por la Disposición Adicional Segunda de la citada Ley, que altera lo preceptuado con carácter general respecto a la facultad de convocatoria de los concursos para cubrir las vacantes de plazas de estos funcionarios, el nombramiento de los mismos, el baremo en la fijación de méritos y la formación de tales funcionarios.

Junto a estas consideraciones destacó el desconocimiento del juego del principio de autonomía local por parte de la Sentencia del Tribunal Constitucional de 7 de abril de 1983, al resolver un conflicto de competencias partiendo de la correlación Estado-Comunidades Autónomas y no del alcance de la intervención de las Entidades locales afectadas en el procedimiento para la provisión de puestos de trabajo en cuanto derivación del principio citado; desconocimiento que ha venido a solucionar la Disposición Adicional Segunda 8, que refuerza el principio de autonomía local al fijar en un 25 por ciento la participación de las Corporaciones locales en la determinación 
de los méritos para el concurso de provisión de plazas de estos funcionarios.

Respecto a la segunda parte, los funcionarios propios de las Corporaciones locales, en primer lugar constató la mayor posibilidad de intervención para la legislación de desarrollo de las Comunidades Autónomas y la existencia de un ámbito mucho mayor de disponibilidad de las Corporaciones locales sobre sus propios funcionarios.

A continuación se refirió al tema de la movilidad de los funcionarios, señalando cómo la regulación del artículo 17,2 , de la Ley $30 / 1984$, excluye la posibilidad de que los funcionarios locales puedan desempeñar puestos de trabajo en la Administración estatal, salvo en el caso previsto por la Disposición Final 3 de la Ley de Bases de Régimen local.

Por último, y respecto a las disposiciones comunes a los funcionarios de carrera, el ponente centró su atención en los preceptos de la Ley de Bases de Régimen local (arts. 95 a 97) dedicados a la participación de los funcionarios en la determinación de sus condiciones de empleo y a la publicidad de las convocatorias de acceso a la Función Pública local, respectivamente, señalando la problemática compleja existente en el primer tema y la singularidad del segundo a través del análisis de las Sentencias del Tribunal Constitucional de 27 de julio de 1982 y 25 de octubre de 1983 relativas a los citados temas.

\section{SEGUNDA SESION}

Dedicada ésta a las «Experiencias puntuales» realizadas en el Estado autonómico. La primera ponencia fue la titulada: «La Ley de la Función Pública de la Administración de la Generalidad», expuesta por el Director General de la Función Pública de la Generalidad de Cataluña, Juan María RAMíREZ CARDÚS.

El punto de partida fue admitir la existencia de posibilidades de desarrollo autonómico de la Función Pública, a pesar de la imperfección de la Ley 30/1984, considerando a ésta como algo absolutamente necesario, pero insuficiente, especialmente para algunas autonomías. La insuficiencia radica en hablar de reforma de la Función Pública sin plantearse la reforma de la Administración, puesto que aquélla es un subsistema de ésta.

A continuación destacó los principales problemas que presenta 
esta Ley: a) una necesidad de desarrollar rápidamente las Leyes autonómicas; b) La preocupación de querer hacerlo demasiado deprisa en el campo de los funcionarios; c) necesidad de una absoluta voluntad política de respetar las Leyes que vamos haciendo.

El paso siguiente fue la exposición de la génesis de la Ley de la Función Pública de la Administración de la Generalidad, aprobada el 23 dejulio de 1985 y publicada en el Boletín Oficial del Estado, número 206 , de 28 de agosto, para exponer seguidamente los criterios fundamentales de esta Ley, que consinderó como una opción, y son los siguientes:

1) Tratamiento interdepartamental de toda la política de personal a través de la Comisión Técnica de la Función Pública, en orden a conseguir que todos los temas de personal sean discutidos por todos los departamentos.

2) Aceptación de la estructura de Cuerpos y Escalas, elementos vertebrales que pueden ayudar a los proceso selectivos y de concurso, aceptando igualmente la posibilidad de personal laboral para determinados puestos.

Destacó asimismo la admisión de la existencia de Cuerpos cuando se trate de desempeñar funciones homogéneas, descritas en el artículo 19, b), y la intención de limitar los Cuerpos en cuanto al número.

3) Respecto a la selección de personal, el trabajo se ha descargado en la Escuela de Administración Pública de Cataluña, la cual interviene directa o indirectamente en todos los órganos de selección, creándose un proceso bifásico de selección para los niveles superiores: el acceso a la propia Escuela y el Curso de Formación en el que se pretende reproducir las condiciones de trabajo del puesto a desempeñar.

En cuanto a los Cuerpos facultativos, el ingreso en la Escuela ha de venir determinado por su anterior preparación profesional, desarrollándose en el Curso la formación básica funcionarial y la específica de aplicacion por estos Cuerpos.

4) Respecto a la carrera administrativa, se ha intentado ofrecer una auténtica carrera a casi todos los colectivos funcionariales.

La fórmula seguida ha sido concebir ésta en tres niveles:

a) Promoción horizontal, que puede llevar a una promoción interna dentro del mismo grupo si existen diferentes escalas. 
b) Profesionalización de la línea de mando. Hasta el nivel de Jefe de Servicio el acceso es por concurso.

Como novedades son destacables:

- La elaboración de un informe normalizado anual o bianual respecto a los funcionarios que ocupen puesto de mando, como médio valorativo para el sistema de concurso.

- La previsión de un expediente administrativo del que se dará audiencia del interesado y cuenta al Comité de Personal para efectuar el cese de los cargos cubiertos por concurso: Jefe de Negociado, Sección y Servicio.

c) Sistemas de oposición o concurso para el acceso a los Cuerpos y Escalas de la Generalidad.

5) Respecto a la participación del personal, se pretende dar validez a las posibles aportaciones técnicas de los funcionarios, los sindicatos, etc., puesto que los intereses de la propia institución no tienen por qué ser contrarios a los intereses de los funcionarios.

6) Respecto a los funcionarios interinos y transferidos, se ha pretendido posibilitar la consolidación de su posición a través de oposiciones restringidas.

7) Por último, y respecto a la movilidad, la postura ha sido considerar que todo aquel que sirva a la Comunidad es funcionario de la misma, salvando las diferencias psicológicas entre el funcionario propio y el transferido, a través del artículo 29 , que reconoce la integración de los funcionarios transferidos y el respeto de sus derechos anteriores, y del artículo 60, que prevé la integración de los mismos en los Cuerpos y Escalas de la Generalidad, así como la adquisición de la condición de funcionarios de ésta.

La segunda ponencia fue la titulada: «El proceso de formación de la Ley de Función Pública valenciana», presentada por el Director del Instituto Valenciano de Administración Pública, Andrés MOREY JUAN.

Comenzó el ponente señalando los presupuestos políticos y técnicos Cel programa electoral del PSOE valenciano, los cuales iban. a condicionar cualquier Anteproyecto de Ley de la Función Pública valenciana, para exponer a continuación la génesis y los principios básicos del Anteproyecto de Ley y concluir con el análisis de los problemas de desarrollo de la Ley. 
Respecto a la génesis y principios básicos, expuso como cuestión previa el proceso reflexivo efectuado en la redacción del Anteproyecto sobre el concepto de funcionario y el de personal laboral, así como la solución adoptada por la Ley.

El estudio de la definición del concepto de funcionario público vino obligado como medio de solución a la dicotomía personal laboral o Función Pública, producida como consecuencia de las diversas posiciones del programa del PSOE y de las fuerzas políticas socialistas que conocen el primer borrador.

En este estudio se llegó a la conclusión de que la característica más propia del concepto de funcionario es la de la permanencia en el trabajo, observando cómo las definiciones de funcionario han incluido esta característica reconduciendo los demás problemas al sometimiento al Derecho administrativo de su relación jurídica.

La solución en la Ley valenciana fue una opción clara hacia el régimen funcionarial, basándose la nueva Administrción en un presupuesto por programas y permitiendo el sistema de clasificación de puestos que se establece, la posibilidad de que puedan calificarse como laborales puestos de trabajo de carácter administrativo que se prevea que no son permanentes o se dude si lo serán.

Advertida esta cuestión previa, a continuación analizó los principios básicos del Anteproyecto que afectan a la Ley.

El primero de ellos es la tendencia a la Función Pública única, cuyas consecuencias son:

- Aplicación de un único régimen jurídico a todos los funcionarios.

- Aplicación de los mismos criterios organizativos y estatutarios por todas las Administraciones de la Comunidad Valenciana.

- Movilidad funcionarial.

- Posibilidad de selección de los funcionarios locales por la Administración autonómica.

El segundo es la consideración del puesto de trabajo como base organizativa, siendo la técnica de clasificación de puestos de trabajo un instrumento esencial para conseguir la eficacia del sistema, que, no obstante, manifestaba problemas prácticos.

El sistema selectivo tradicional se mostraba como el primer obstáculo al sistema puro de puestos de trabajo.

El tercero de los principios básicos es el sistema de selección de personal. 
Tras analizar los cambios que ha sufrido el concepto de selección ante la movilidad predicada en la Ley 30/1984, y dudar de la utilidad del sistema selectivo de esta Ley en un sistema de puestos de trabajo o en una Administración que no necesite hacer selecciones masivas de personal, expuso el sistema de habilitación del Anteproyecto.

En éste se pretendió establecer un sistema polivalente para el ingreso y para el acceso en puestos de libre designación, que se denominaba sistema de habilitación. Este sistema suponía la división del proceso en dos fases: la primera era la habilitación propiamente dicha, y la segunda, el concurso para proveer cada puesto de trabajo. La primera no confería ninguna relación jurídica con la Administración. Era la segunda la que consolidaba esta relación.

Sin embargo, la Ley de la Función Pública valenciana sólo ha conservado la denominación del proceso selectivo como "prueba de habilitación» y la distinción de dos fases: la selección propiamente dicha y el acceso al puesto concreto.

Por último, analizó los problemas de desarrollo de la Ley de la Función Pública valenciana respecto de los principios básicos señalados, y apuntó las posibles soluciones en situaciones determinadas.

La tercera ponencia, titulada "Problemas y perspectivas de la organización de la Administración y la Función Pública de las Comunidades Autónomas: el caso de Aragón», fue presentada por el Secretario General Técnico del Departamento de Presidencia y Relaciones Institucionales de la Diputación General de Aragón, Manuel JIMÉNEZ ABAD.

Comenzó afrontando la nueva organización del Estado establecida por la Constitución de 1978, señalando la exigencia de un replanteamiento del sistema de Administraciones públicas, siendo ahora la reforma administrativa más necesaria que nunca.

A continuación y tras diferenciar la reforma de la Administración pública de la reforma de la Función Pública, describió el marco general en el que se incribe cualquier acción politica y administrativa sobre las Administraciones de las Comunidades Autónomas, marco que se caracteriza por un auténtico deterioro institucional de la Adninistración del Estado y por los problemas derivados de los defectos de planeamiento en la construcción del Estado autonómico, que han dificultado la organización de las Administraciones públicas de las Comunidades Autónomas.

En este marco, y para conseguir el objetivo de efectuar un dise- 
ño general de lo que se deseara que fuera la Administración pública de Aragón, se elaboró un informe y una propuesta de directrices por el Gobierno de Aragón, que fue aprobado unánimemente por las Cortes de Aragón.

La referencia general a los principales problemas que plantea este informe fue el contenido básico de la ponencia, cuyas líneas fundamentales fueron las siguientes:

1. En primer lugar destacó como básica la cuestión de la organización administrativa.

Realizando una consideración previa, señaló la importancia de un "órgano de organización» central suficientemente apoyado en sus decisiones y la necesaria coordinación de la unidad de organización con la unidad de presupuestos del Departamento de Hacienda. Seguidamente hizo referencia a los problemas básicos que plantea la organización administrativa de las Comunidades Autónomas:

a) Necesidad de que en su organización se produzca una integración suficiente entre las diversas unidades que la componen.

b) Huir de la petrificación de las organizaciones públicas.

c) Ajuste de la organización administrativa a la realidad competencial y a las particulares dimensiones territorial y personal de cada Comunidad Autónoma.

d) Ha de existir una integración de la Administración a través de la vertebración horizontal.

e). Necesidad de delimitación entre órganos políticos y administrativos.

f) Necesidad de reflexión sobre el tratamiento a dar a los servicios periféricos de las Administraciones autonómicas, especialmente sobre los problemas que presenta el modelo de articulación de los servicios periféricos propios a través de las Diputaciones provinciales.

g) La posible introducción de unidades orgánicas cuyo ámbito territorial de actuación sea inferior al de la Provincia, es decir, servicios comarcales.

h) Necesidad de evitar radicalmente la proliferación de organismos autónomos en el seno de las Administraciones autonómicas. 
2. En segundo lugar analizó como básico el funcionamiento de la Administración.

Poniendo de relieve la inadaptación de nuestra Administración a las modernas técnicas de gestión y criterios de organización respecto a las organizaciones privadas, advirtió la ocasión de incorporar nuevos modelos, nuevas técnicas a la organización administrativa de las Comunidades Autónomas.

Para la construcción de las Administraciones autonómicas propuso como principios de actuación a introducir en el funcionamiento de las mismas los siguientes:

a) Planificación y programación a todos los niveles en las Administraciones autonómicas necesariamente coordinada con la programación económica y presupuestaria.

b) Coordinación de funciones y correcto funcionamiento de los órganos colegiados que tengan funciones coordinadoras.

c) Desconcentración de funciones en la organización periférica de las Comunidades Autónomas.

d) Productividad y control de eficacia de las actuaciones conectada con la modificación de los criterios tradicionales de inspección de servicios.

e) Necesidad de una valoración de la introducción de la informática en el funcionamiento de la Administración y estudio de reasignación de efectivos auténticos.

3. El tercero y último tema básico fue el análisis de la Función Pública a través de la determinación de las principales caracteristicas y principios del Proyecto de Ley Ordenadora de la Función Pública de Aragón.

Estos principios son los siguientes:

a) Principio de unidad de acción en la política de personal.

b) Principio de simplificación de las estructuras, creándose un Cuerpo por cada nivel de titulación.

c) Las relaciones de puestos de trabajo como clave del sistema.

d) La oferta anual de empleo público como consecuencia de unos procedimientos internos de provisión de puestos de trabajo y antecedente de los procedimientos de selección.

e) Reforma del sistema selectivo general introduciendo nuevas fórmulas en selección, provisión y carrera administrativa. 


\section{TERCERA SESION}

Estuvo dedicada a las «Experiencias funcionariales en EuskalHerria", tema sobre el que se presentaron tres ponencias.

La primera, titulada «La Función Pública en Navarra: evolución», estuvo a cargo del Profesor de Derecho Administrativo de la Universidad del Pais Vasco, Demetrio LOPERENA RoTA.

Comenzó con una introducción, en la que destacó la singularidad del régimen navarro y el interés juridico que presenta el estudio de esta singularidad.

A continuación expuso los principales caracteres de la Función Pública navarrả del siglo XIX y su evolución a través del análisis de la legislación de este periodo enlazando con el Estatuto provincial de 20 de marzo de 1920 , que garantizaba los funcionarios forales, y el Decreto-ley de 4 de noviembre de 1925, que estableció las bases para el Estatuto municipal de Navarra, bases desarrolladas por el. Reglamento de Administración municipal de 3 de febrero de 1928, que mantiene su vigencia en algunos aspectos.

La conclusión extraída del estudio de éste periodo anterior a la transición política fue la constatación de la inexistencia de problemas excesivos en cuanto a la relación de la legislación específica navarra con la legislación general.

Seguidamente advirtió, como caracteristicas de la década del setenta, la existencia de disposiciones que pretenden adaptar la estructura administrativa y personal, así como normas que tratan de incorporar los nuevos derechos y libertades al régimen funcionarial navarro.

En la exposición de la normativa vigente, tras señalar cuál es el fundamento estatutario y el contenido de la Ley Foral 13/1983, de 30 de marzo, Estatuto actual de la Función Pública navarra, puntualizó como caracteres más sobresalientes de esta Ley los siguientes:

a) Ordenación sistemática y globalizante, que sustituye a la normativa asistemática precedente.

b) Aceptable racionalización de niveles y grados.

c) Unificación de los regímenes jurídicos para los funcionarios locales y forales.

d) Adecuación de la forma de acceso a los principios constitucionales de mérito y capacidad. 
e) Desaparición absoluta de las prebendas.

f) Creación de la Escuela de Funcionarios Públicos.

g) No se hace previsión legal sobre el personal que se transfiera de la Administración central.

h) Actual desarrollo reglamentario de la misma en su casi totalidad de materias.

Como punto previo a las conclusiones finales advirtió la problemática de la relación entre la legislación foral y la legislación general en materia de funcionarios.

En este tema, por una parte, atribuyó a la Disposición Adicional Primera de la Constitución, la cual ampara y reconoce el respeto a los derechos históricos, el carácter de título jurídico, que permite a las Cortes excepcionar el régimen jurídico funcionarial para $\mathrm{Na}$ varra, y, por otra parte, al examinar el alcance de la legislación estatal sobre el régimen funcionarial navarro, postuló su inaplicación siempre que se encuentre en contradicción con este régimen.

Como punto final de su exposición llegó a las siguientes conclusiones:

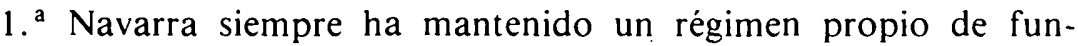
cionarios regulado por normas emanadas de sus instituciones.

$2 .^{a}$ Los medios técnicos de articulación han sido disposiciones excepcionales.

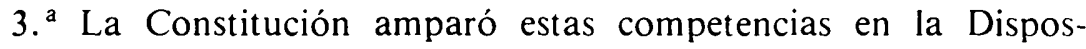
ción Adicional Primera.

4. ${ }^{a}$ La Ley Foral navarra $13 / 1983$, de 30 de marzo, no está sometida a las bases estataleś.

5. ${ }^{\text {a }}$ Esta Ley supone una ordenación sistemática y globalizante de la Función Pública.

6. ${ }^{a}$ La ambigüedad de las disposiciones de excepción: Disposición Adicional Primera de la Constitución, Disposición Adicional Decimocuarta de la Ley 30/1984 y Disposición Adicional Tercera de la Ley $7 / 1985$, suponen un reenvío al compromiso político ulterior, sistema que puede llevar a una aproximación de la excepcionalidad a la generalidad.

La segunda ponencia, titulada «La Función Pública local de Euskadi», fue expuesta por el Catedrático de Derecho Administrativo de la Universidad del País Vasco, José Manuel CASTElls ARTECHE. 
Como punto de partida de su exposición acotó el ámbito de la misma a la Función Pública foral y local.

A continuación trató la evolución histórica del tema a través del examen de diversos periodos: la Restauración, la década de 1920, la II República y la Constitución de 1978, señalando las principales caracteristicas de cada uno y advirtiendo cómo la configuración auténtica de la Administración actual se produjo en la década de 1920.

El siguiente paso fue el examen de las notas caracteristicas de la Función Pública vasca en el contexto derivado de la Constitución de 1978. Destacó como rasgos principales, por una parte, una anticipación progresista respecto a la regulación básica estatal de la Función Pública $\mathrm{y}$, por otra, un enfrentamiento reiterado con las autoridades centrales y plasmado en los diversos conflictos de competencias resueltos por el Tribunal Constitucional en esta' materia.

Como consecuencia de este examen, advirtió una indeterminación del marco general de la Función Pública y una indeterminación del marco doméstico de la Función Pública vasca, ya que en la Ley de Territorios Históricos no hay referencia alguna expresa a los funcionarios locales.

En este marco de indeterminación concretó la aprobación de la Ley de Bases de Régimen local, de cuya Disposición Adicional Segunda afectuó un nuevo análisis y apuntó su carácter de vía de salida a los conflictos anteriores utilizando la cobertura normativa de la Disposición Adicional Primera de la Constitución.

En el análisis del sistema de la Disposición Adicional Segunda de la Ley de Bases de Régimen local, que calificó de complejo, advirtió dos cuestiones materiales de alcance.

En primer lugar, la primicia y avance dinámico que supone la consagración legal de la Disposición Adicional Primera de la Constitución como fundamento de la especialidad del régimen funcionarial vasco, materia que en el Estatuto de Autonomía no se encuentra relacionada ni apoyada en tal Disposición Adicional.

Asimismo destacó la gran importancia, de cara a la aparición de los Estatutos de la Función Pública estatal y autonómicos, de la opción asumida por el legislador de cubrir las especialidades funcionariales vascas con la Disposición Adicional Primera de la Constitución (frente a cierta doctrina que regula el carácter de Derecho histórico a la cuestión del funcionariado).

En segundo lugar, y destacando el papel que desempeñan los 
territorios forales en la Disposición Adicional Segunda, cuestionó, por un lado, la equiparación entre Derechos históricos y territorios forales $y$, por otro, el grado de incidencia de esta Disposición Adicional en los principios inspiradores de la Ley de Territorios Históricos.

Como conclusión de este análisis, destacó la ocupación por las instituciones forales del ámbito de actuación de las instituciones comunes, con la consecuente potenciación del papel a desempeñar por aquéllas.

Por último, señaló la necesidad de un reconocimiento de la especificidad del régimen funcionarial vasco, tanto por la Ley estatal de Función Pública como por la Ley vasca, siendo indispensable en esta última una regulación homogénea y pareja de la Función Pública local y foral.

La tercera ponencia, que versó sobre «Rasgos generales de la Función Pública vasca», fue presentada por el Viceconsejero de Administración y Función Pública del Gobierno Vasco, José Ignacio IGLESIAS LEZAMA.

El contenido de la exposición fue exclusivamente el análisis de la Administración general e institucional, de acuerdo con las coordenadas estatales, prescindiendo de la Administración local y foral.

Este análisis fue realizado a través del tratamiento de tres grandes apartados.

El primero de ellos se refirió al examen del proceso de institucionalización junto con el marco normativo estatal de referencia.

En este proceso destacó, por una parte, la normativa dictada por el Gobierno Vasco al amparo de la Disposición Transitoria Segunda del Estatuto de Autonomía, normativa relativa a contratación de personal y organización administrativa principalmente, sin olvidar la regulación de materias particulares como las referidas a incompatibilidades, derechos pasivos y reglamentaciones de carácter interno.

Por otro lado, y haciendo referencia al marco normativo estatal, examinó la incidencia de esta legislación - Ley 12/1983, de 14 de octubre, del Proceso Autonómico; Ley 30/1984 de Medidas para la Reforma de la Función Pública; Ley 7/1985 de Bases del Régimen 1. cal- en la construcción de la Administración vasca y con relación a la Ley 27/1983 del Parlamento vasco de «relaciones entre las insti- 
tuciones comunes de la Comunidad Autónoma y los órganos forales de sus territorios históricos».

El segundo apartado se centró en la exposición de las caracteristicas actuales del personal y de los órganos de la Administración vasca.

Respecto a las caracteristicas del personal, efectuó un análisis estadístico pormenorizado de la composición y cuantificación del personal al servicio de la Administración autonómica, cuya conclu- sión fue la constatación de la diversidad de origen y vínculo jurídico del conjunto del personal de esta Administración.

Por lo que hace referencia a los órganos de la Administración vasca, advirtió la importancia del Departamento de Presidencia y Justicia como órgano superior jerárquico competente en materia de Función Pública vasca, de acuerdo con la determinación de funciones y contenido competencial efectuado por el Decreto 101/1984 del Gobierno Vasco $(*)$.

La tercera y última cuestión examinada por el ponente fue el tratamiento de las orientaciones futuras de la Función Pública vasca.

En primer lugar, consideró como premisas básicas para cualquier actuación normativa posterior las siguientes:

a) La necesaria diferenciación entre Administración como sistema de gestión y como conjunto institucional de personas que la componen.

b) Asunción de los objetivos a cubrir y directrices a seguir, tanto por la Administración como por las pèrsonas a quien van dirigidas.

A continuación y considerando al puesto de trabajo como elemento central del sistema, señaló las líneas de actuación del Gobierno Vasco, asi como los proyectos específicos para la ordenación de la Función Pública vasca, que principalmente son los siguientes:

- Reconocimiento del euskera y potenciación de su uso por el personal de la Administración vasca.

(*) Derogado por el Decreto 5/85, de 27 de enero (BOPV, núm. 21. de 28-I-85), sobre determinacion de funciones y areas de actuacion del Gobierno Vasco. 
- Existencia de un funcionariado preparado de cara a las relaciones con la CEE.

- Adecuación de los procesos de ingreso, selección y formación de los funcionarios a los principios de igualdad, mérito y capacidad.

- Formación del funcionariado a cargo del Instituto Vasco de Administración Pública.

- Extinción del personal contratado e interino.

- Predominio del concepto de puesto de trabajo.

- Determinación de los puestos de trabajo laborales.

- Definición de los órganos de la Función Pública vasca.

- Fijación de las incompatibilidades.

- Reciclaje de efectivos.

Como resumen, señaló la intención de flexibilizar las estructuras administrativas, conseguir una profesionalización del funcionariado y llegar a una eficaz prestación de los servicios.

\section{CLAUSURA}

El acto de clausura de estas densas e intensas Jornadas fue presidido por el Consejero de Presidencia y Justicia del Gobierno vasco, Juan Ramón Guevara SALETA.

En breves palabras advirtió la necesidad de una Administración ágil, eficaz y bien organizada de las Comunidades Autónomas como requisito fundamental para la construcción del Estado de las Autonomías, el cual responde a una demanda de autogobierno, cuya consecuencia ha de ser la existencia de una Función Pública propia.

Asimismo expresó el reto pendiente del Gobierno Vasco, que no es otro que hacer que la Administración vasca funcione y se consolide como tal, para lo cual consideró como fundamental la existencia de una única Función Pública vasca.

\section{CONCLUSIONES FINALES}

Una vez realizada la aproximación al contenido de las ponencias presentadas por los protagonistas principales de estas Jornadas, sólo me resta realizar una breve valoración y balance de las mismas. 
En primer término, es preciso destacar la crítica directa y contundente recibida por la Ley 30/1984 de Medidas para la Reforma de la Función Pública, cuyos principales detractores fueron los Catedríticos de Derecho Administrativo de la Universidad de Alcalá de Henares y Complutense, Alejandro NIETO y Tomás Ramón FERNÁNDEZ, respectivamente.

Como asistente a estas Jornadas, la sensación que pude experimentar fue, por una parte, la apreciación del reiterado y criticado panorama de la Función Pública estatal y, por otro, la constatación del interés y aspiraciones de las Comunidades Autónomas allí representadas, en orden a la recuperación y distanciamiento del "caos» administrativo estatal para conseguir - dentro y a partir del escaso margen de actuación dejado a las mismas por la Ley 30/1984- una estructuración racional de su Función Pública al margen de los defectos y lacras de la Función Pública estatal.

Por otro lado, no hay que dejar en el olvido la predisposición de los ponentes y participantes a buscar salidas para el tren de la Función Pública, que todavía se encuentra en un largo y oscuro túnel.

En otro orden de cosas, es preciso advertir el valioso contraste de las aportaciones y modelos de Función Pública de las distintas Comunidades Autónomas que ya han regulado la materia, como punto de referencia y ayuda a las futuras regulaciones normativas a realizar por las restantes.

El balance final de estas Jornadas puede considerarse muy positivo. Desde el punto de vista organizativo, hay que destacar la oportunidad de su iniciativa, que surgida conjuntamente del Instituto Vasco de Administración Pública y de la Facultad de Derecho de la Universidad del Pais Vasco ha llegado a buen puerto, puesto que la conjunción y colaboración de la Universidad con la Administración ha dado como resultado una serie de consideraciones, críticas, modelos e ideas vertidas durante la celebración de estas Jornadas, que pueden significar un paso adelante en el futuro y necesario análisis reflexivo previo a cualquier proceso de estructuración y reforma de la Función Pública; proceso que inexcusablemente habrá de realizarse en torno a la futura Ley de Función Pública de la Comunidad Autónoma de Euskadi.

Como punto final, sólo queda expresar la esperanza impaciente 
por la pronta publicación de las ponencias de estas Jornadas a cargo del Instituto Vasco de Administración Pública, publicación que va a ser indispensable para el estudio y conocimiento de la deseada Función Pública autonómica. 


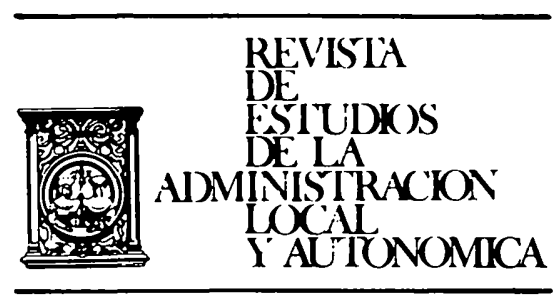

III. JURISPRUDENCIA

REALA-1986, núm. 229. PEREZ BARRIO, AGUSTIN. JORNADAS DE.ESTUDIO SOBRE ADMINISTR... 
REALA-1986, núm. 229. PEREZ BARRIO, AGUSTIN. JORNADAS DE ESTUDIO SOBRE ADMINISTR...

REALA-1986, núm. 229. PEREZ BARRIO, AGUSTIN. JORNADAS DE ESTUDIO SOBRE ADMINISTR... 